\title{
The clinical and economic burden of non-alcoholic steatohepatitis in the Middle East: behind the scene
}

\author{
Lung-Yi Mak $^{1,2} \cdot$ James Fung ${ }^{1,2}$ (1)
}

Received: 12 April 2021 / Accepted: 25 May 2021 / Published online: 18 June 2021

(c) Asian Pacific Association for the Study of the Liver 2021

Non-alcoholic fatty liver disease (NAFLD) is the most common cause of chronic liver disease worldwide, with the Middle East having one of the highest prevalence rate of NAFLD among all other areas of the world [1]. Similar to the Western countries, non-alcoholic steatohepatitis (NASH) has become the leading primary disease indication for liver transplantation. With the growing prevalence of obesity and diabetes mellitus, which are both commonly coexisting with NAFLD, it is projected that the prevalence of NAFLD in Saudi Arabia, one of the main countries in the Middle East, will rise from $25.7 \%$ in year 2017 to $31.7 \%$ in year 2030 [2]. Based on estimates from Saudi Arabia, United Arab Emirates and Kuwait, together with published data of disease progression from USA patients, Sanai et al. [3] used Markov models to project the estimated prevalence of different disease phase of NAFLD (including different stages of fibrosis, compensated cirrhosis, decompensated cirrhosis, hepatocellular carcinoma [HCC], liver transplantation and death) from 2018 to 2030 . They calculated the direct health care costs associated with NASH, including medications, investigations, consultations, hospitalization, procedures and liver transplant. The alarmingly high predicted prevalence of different disease phases of NAFLD together with the unsurprisingly huge proportion of health expenditure on patients with NASH call for necessary preventive measures to avoid this from becoming real in the year 2030. The message is clear and applicable to all countries affected by the pandemic of obesity and NAFLD.

There are no published data of disease progression rates in the natural history of NAFLD specific to the Middle East

James Fung

jfung@gastro.hk

1 Department of Medicine, Queen Mary Hospital, The University of Hong Kong, Pokfulam Road 102, Hong Kong, China

2 State Key Laboratory of Liver Research, The University of Hong Kong, Hong Kong, China population. The authors calculated the disease phase transition probabilities based on Alswat et al. [2], which was in fact based on USA data [4]. It has been well reported that genetic factors play a significant role in disease progression in NAFLD. For instance, the PNPLA3 gene polymorphism is associated with NASH and fibrosis, and the phenotype demonstrates inter-ethnic differences [5]. Inter-racial differences are also observed for the loss-of-function splice variant of the gene encoding hydroxysteroid 17-beta dehydrogenase 13 (HSD17B13) that confers protection from advanced liver disease in NAFLD [6]. Based on the genetic factors and differences in lifestyle between different ethnic subgroups, assumption of disease progression rates in the Middle East population using USA data may not be entirely accurate. Also, the prevalence data of NAFLD in the Middle East was based on ultrasonographic diagnosis without histological data. Despite these limitations which are unlikely to be addressed within a short time, the authors' effort to arouse public awareness and to inform policy makers about this important non-communicable disease is timely and important.

When looking into the direct health care costs of NAFLD, there are two important components to consider. First, the main bulk of health care expenditure is used on patients with milder form of NAFLD (F0-F3) due to the fact that these patients comprise the majority in this disease entity (Fig. 1). While the investigations mentioned by the authors (Fibroscan, Liver function test, hep B/C serology, autoimmune liver screen, etc.) are reasonable, it is a good opportunity to evaluate whether we are over-investigating/over-treating (e.g., dietitian referral for patients with $\mathrm{F} 0-\mathrm{F} 1$ ) patients with normal liver function parameters and milder forms of the disease. Stepping down to primary care is a good strategy to minimize the health care cost associated with specialist visit that is significantly more expensive than consultation with a general practitioner in most countries of the Middle East and the rest of the world. After more than a decade of debate, a recent consensus from an international panel recommended 


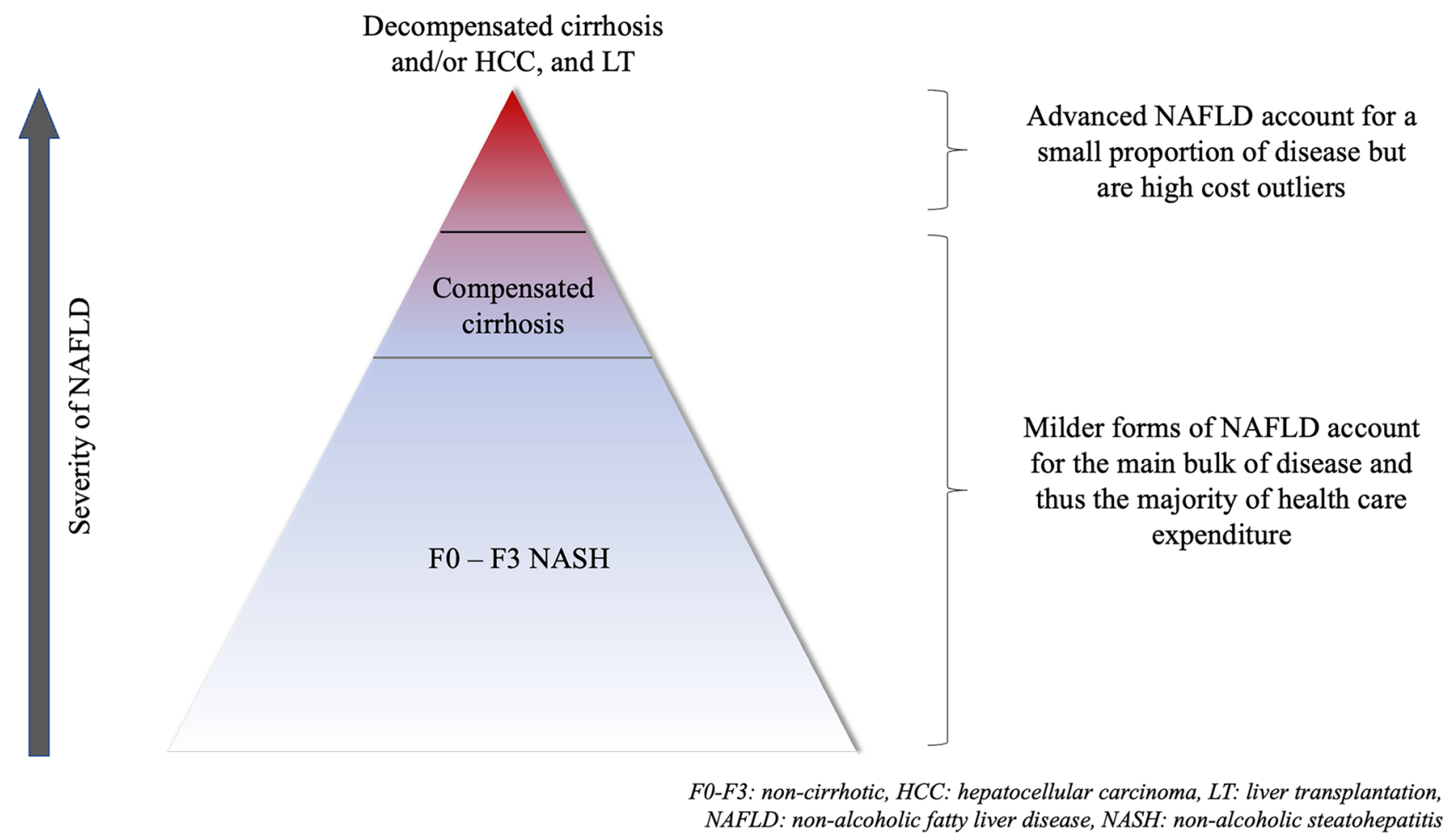

Fig. 1 Schematic diagram illustrating the contribution to health care cost expenditure in patients with NAFLD

a new definition of fatty liver disease into metabolic dysfunction-associated fatty liver disease (MAFLD) using positive diagnostic criteria, which more accurately reflect the disease pathogenesis and help in patient stratification for management [7]. By adopting the proposed simplified diagnostic algorithm of MAFLD, it is expected that the task of provision of basic care can be shifted to general practitioners and allied health professionals for patients at low risk, while priority for specialist care will be given to those at high risk of hepatic and extra-hepatic complications [8].

Second, like many chronic medical illnesses such as inflammatory bowel disease (IBD) [9], there are high-cost outliers that account for a significant proportion of expenditure. In the NAFLD population, high-cost outliers are those with HCC or decompensated cirrhosis that may eventually require liver transplantation. The identification of high-cost outliers theoretically allows early intervention to halt disease progression and reduce subsequent expenditure on managing the complications of decompensated cirrhosis or HCC. However, unlike IBD, there are no approved pharmacological treatment at the moment that can serve that purpose. The most effective treatment would be achieving significant weight loss $(\geq 7 \%)$ that can potentially lead to regression of fibrosis and prevent further disease progression. Unfortunately, up to $50-70 \%$ of patients failed to achieve target weight loss [10]. Being easier said than done, the advocacy of promoting weight loss in NAFLD or obese people requires efforts from multiple dimensions of the health care system and the society. Additional interventions to motivate patients for behavioral changes regarding diet and physical activity will be necessary to aid NAFLD patients reach their target weight loss. Recently, the World Health Organization recommends digital health intervention in the form of digital tracking of clients' health status and services combined with decision support. This is a good example of promoting weight loss using alternative strategies than routine physician counselling.

Apart from the direct health care costs related to management of liver disease, other costs should also be taken into consideration, especially cardiovascular diseases which account for the most common cause of mortality in NAFLD patients [1]. Cardiovascular disease-related costs are no less than liver disease-related costs and bring tremendous burden to the health care system [11]. Moreover, as the authors also addressed, indirect costs arising from the diagnosis of NAFLD also bring significant economic impact. These include loss of efficient working days from absenteeism (outpatient visits or hospitalization leading to sick leave from work), presenteeism (impact of health problems while on duty for work), and premature mortality from the disease [12].

Sanai et al. calls for strengthened efforts to alter the trajectory of NAFLD and prevent the alarming scenario of NAFLD affecting one-third of their population coming 
true. The authors identified key areas of focus, including the creation of a national plan for prevention and education, disease surveillance, and access to eventual treatments when these become available. The clinical and economic burden of NAFLD cannot be overlooked at this point in time, and is likely to grow exponentially if the necessary measures are not undertaken in a timely manner. Importantly, the results of this study should also serve as a wake-up call to policy makers in other regions where obesity and diabetes mellitus are rising.

\section{References}

1. Younossi ZM, Koenig AB, Abdelatif D, Fazel Y, Henry L, Wymer M. Global epidemiology of nonalcoholic fatty liver disease-Metaanalytic assessment of prevalence, incidence, and outcomes. Hepatology. 2016;64:73-84.

2. Alswat K, Aljumah AA, Sanai FM, Abaalkhail F, Alghamdi M, Al Hamoudi WK, Al Khathlan A, et al. Nonalcoholic fatty liver disease burden - Saudi Arabia and United Arab Emirates, 2017 2030. Saudi J Gastroenterol. 2018;24:211-9.

3. Sanai FM, Al Khathlan A, Al Fadhli A, Jazzar AS, Hashim AM, Mansour E, Abaalkhail F, et al. Clinical and economic burden of nonalcoholic steatohepatitis in Saudi Arabia, United Arab Emirates and Kuwait. Hepatol Int. 2021. https://doi.org/10.1007/ s12072-021-10182-x (in press)

4. Estes C, Razavi H, Loomba R, Younossi Z, Sanyal AJ. Modeling the epidemic of nonalcoholic fatty liver disease demonstrates an exponential increase in burden of disease. Hepatology. 2018;67:123-33.

5. Zain SM, Mohamed R, Mahadeva S, Cheah PL, Rampal S, Basu RC, Mohamed Z. A multi-ethnic study of a PNPLA3 gene variant and its association with disease severity in non-alcoholic fatty liver disease. Hum Genet. 2012;131:1145-52.

6. Kozlitina J, Stender S, Hobbs HH, Cohen JC. HSD17B13 and chronic liver disease in blacks and hispanics. N Engl J Med. 2018;379:1876-7.

7. Eslam M, Sanyal AJ, George J, International Consensus P. MAFLD: a consensus-driven proposed nomenclature for metabolic associated fatty liver disease. Gastroenterology. 2020;158:1999-2014 e1991.

8. Mendez-Sanchez N, Arrese M, Gadano A, Oliveira CP, Fassio E, Arab JP, Chavez-Tapia NC, et al. The latin American association for the study of the liver (ALEH) position statement on the redefinition of fatty liver disease. Lancet Gastroenterol Hepatol. 2021;6:65-72.

9. Mak LY, Ng SC, Wong IOL, Li MKK, Lo FH, Wong MTL, Leung $\mathrm{CM}$, et al. Direct health-care cost utilization in Hong Kong inflammatory bowel disease patients in the initial 2 years following diagnosis. J Gastroenterol Hepatol. 2018;33:141-9.

10. Vilar-Gomez E, Martinez-Perez Y, Calzadilla-Bertot L, TorresGonzalez A, Gra-Oramas B, Gonzalez-Fabian L, Friedman SL, et al. Weight loss through lifestyle modification significantly reduces features of nonalcoholic Steatohepatitis. Gastroenterology. 2015;149:367-378 e365 (quiz e314-365).

11. Russell MW, Huse DM, Drowns S, Hamel EC, Hartz SC. Direct medical costs of coronary artery disease in the United States. Am J Cardiol. 1998;81:1110-5.

12. Kieu TTM, Trinh HN, Pham HTK, Nguyen TB, Ng JYS. Direct non-medical and indirect costs of diabetes and its associated complications in Vietnam: an estimation using national health insurance claims from a cross-sectional survey. BMJ Open. 2020;10:e032303.

Publisher's Note Springer Nature remains neutral with regard to jurisdictional claims in published maps and institutional affiliations. 